\title{
Article \\ HERV-K Gag RNA and Protein Levels Are Elevated in Malignant Regions of the Prostate in Males with Prostate Cancer
}

\author{
Simin D. Rezaei ${ }^{1,+}$, Joshua A. Hayward ${ }^{1,2}$, Sam Norden ${ }^{3}$, John Pedersen ${ }^{3}$, John Mills ${ }^{1,3,4}$, Anna C. Hearps ${ }^{1,4, \ddagger}$ \\ and Gilda Tachedjian $1,2,5, *, \ddagger(\mathbb{D}$ \\ 1 Life Sciences Discipline, Burnet Institute, 85 Commercial Road, Melbourne, VIC 3004, Australia; \\ sdrezaei@burnet.edu.au (S.D.R.); joshua.hayward@burnet.edu.au (J.A.H.); john.mills@monash.edu (J.M.); \\ anna.hearps@burnet.edu.au (A.C.H.) \\ 2 Department of Microbiology, Monash University, Clayton, VIC 3168, Australia \\ 3 TissuPath Specialist Pathology, Mount Waverley, VIC 3149, Australia; sam.norden@tissupath.com.au (S.N.); \\ john.pedersen@tissupath.com.au (J.P.) \\ 4 Department of Infectious Diseases, Monash University, Melbourne, VIC 3004, Australia \\ 5 Department of Microbiology and Immunology at the Peter Doherty Institute for Infection and Immunity, \\ University of Melbourne, Melbourne, VIC 3010, Australia \\ * Correspondence: gilda.tachedjian@burnet.edu.au; Tel.: +61-3-9282-2256 \\ + Present address: Centre for Integrative Infectious Diseases, Integrative Virology, University Hospital \\ Heidelberg, 69120 Heidelberg, Germany. \\ $\ddagger \quad$ These authors contributed equally to this work.
}

Citation: Rezaei, S.D.; Hayward, J.A.; Norden, S.; Pedersen, J.; Mills, J.;

Hearps, A.C.; Tachedjian, G. HERV-K Gag RNA and Protein Levels Are Elevated in Malignant Regions of the Prostate in Males with Prostate Cancer. Viruses 2021, 13, 449. https://doi.org/10.3390/v13030449

Academic Editor: Ester Ballana Guix

Received: 15 February 2021

Accepted: 8 March 2021

Published: 10 March 2021

Publisher's Note: MDPI stays neutral with regard to jurisdictional claims in published maps and institutional affiliations.

Copyright: (c) 2021 by the authors. Licensee MDPI, Basel, Switzerland. This article is an open access article distributed under the terms and conditions of the Creative Commons Attribution (CC BY) license (https:// creativecommons.org/licenses/by/ $4.0 /)$.
Abstract: Heightened expression of human endogenous retrovirus (HERV) sequences has been associated with a range of malignancies, including prostate cancer, suggesting that they may serve as useful diagnostic or prognostic cancer biomarkers. We analysed the expression of HERV-K (Gag and Env/Np9 regions), HERV-E 4.1 (Pol and Env regions), HERV-H (Pol) and HERV-W (Gag) sequences in prostate cancer cells lines and normal prostate epithelial cells using qRT-PCR. HERV expression was also analysed in matched malignant and benign prostate tissue samples from men with prostate cancer $(n=27$, median age 65.2 years (range $47-70)$ ) and compared to prostate cancerfree male controls $(n=11)$. Prostate cancer epithelial cell lines exhibited a signature of HERV RNA overexpression, with all HERVs analysed, except HERV-E Pol, showing heightened expression in at least two, but more commonly all, cell lines analysed. Analysis of primary prostate material indicated increased expression of HERV-E Pol but decreased expression of HERV-E Env in both malignant and benign regions of the prostate in men with prostate cancer as compared to those without. Expression of HERV-K Gag was significantly higher in malignant regions of the prostate in men with prostate cancer as compared to matched benign regions and prostate cancer-free men ( $p<0.001$ for both), with $85.2 \%$ of prostate cancers donors showing malignancy-associated upregulation of HERV-K Gag RNA. HERV-K Gag protein was detected in 12/18 (66.7\%) malignant tissues using immunohistochemistry, but only $1 / 18(5.6 \%)$ benign tissue sections. Heightened expression of HERV-K Gag RNA and protein appears to be a sensitive and specific biomarker of prostate malignancy in this cohort of men with prostate carcinoma, supporting its potential utility as a non-invasive, adjunct clinical biomarker.

Keywords: human endogenous retrovirus (HERV); prostate cancer; HERV-K

\section{Introduction}

Prostate cancer is the second most common cancer in males worldwide and the second most common cause of cancer deaths amongst males in many high-income countries [1]. Prostate-specific antigen (PSA)-based screening for prostate cancer was introduced in the late 1980s and has since contributed substantially to early disease detection and the subsequent decline in age-standardised mortality rates [1]. Five-year survival rates for 
prostate cancer in many settings are now $>90 \%$, with some countries achieving early diagnosis in over $80 \%$ of cases in either stage I or II [2]. However, one potential consequence of improved detection methods may be the "over-diagnosis" and treatment of clinically insignificant prostate cancers, with a recent analysis suggesting that $42 \%$ of prostate cancers in Australia may have been over-diagnosed [3]. A greater understanding of additional, measurable parameters that may facilitate the identification of clinically relevant prostate cancers would be beneficial to prevent physical and psychosocial damages associated with over-diagnosis and treatment.

Human endogenous retrovirus (HERV) sequences constitute $8 \%$ of the human genome and represent the remnants of ancestral germline infections by retroviruses previously capable of inter-individual (exogenous) transmission [4]. Although substantial deletions and inactivating mutations have rendered these viruses non-infectious, HERV elements can be transcriptionally active and many sequences retain open reading frames capable of producing viral proteins [5]. Furthermore, HERV regulatory elements located in viral long terminal repeat (LTR) regions can provide tissue-specific enhancers, promoters or alternative polyadenylation signals that may influence the expression of nearby genes [6,7].

There are approximately 40 families of HERVs characterized in the human genome, with the most recently acquired family, HERV-K, retaining the greatest number of intact viral genes [8]. Whilst HERV gene transcription is largely suppressed by epigenetic mechanisms, altered expression of HERV elements is associated with a range of conditions, including neurological diseases, multiple sclerosis, autoimmune conditions, diabetes and, probably most prominently, cancer [3,9]. Enhanced expression of HERV elements through detection of RNA transcripts and viral proteins occurs in a range of cancer types, including breast, melanoma, colorectal and prostate cancers (for recent review see Reference [8]). Malignancy is also associated with increased levels of antibodies to HERV proteins, suggesting that these factors can be immunogenic $[10,11]$ and may represent potential therapeutic targets. Importantly, from a prognostic point of view, a number of studies report associations between heightened HERV activity and disease severity, metastasis and survival [12-14].

With respect to prostate cancer, previous studies have reported elevated expression of HERV-K and HERV-E RNAs in malignant as compared to normal prostate tissue [15-17] and in peripheral blood mononuclear cells (PBMC) of men with prostate cancer [18]. However, substantial variation exists regarding the extent of HERV element upregulation observed in individuals with prostate cancer [15]. This, combined with the observation that factors such as ethnicity and age can affect the expression of HERV mRNA [18], highlights the need to validate HERV expression as a prostate cancer biomarker in different clinical and demographic settings.

In this study, we assessed the expression level of HERV transcripts in matched benign and malignant prostate tissues from men with prostate cancer and compared this expression to prostate cancer-free men. We report evidence of both global increases in HERV transcript expression in prostate tissues of men with prostate cancer as well as heightened expression of HERV-K transcripts specifically in malignant prostate tissue. These findings may be relevant for the development of diagnostic and prognostic biomarkers for prostate cancer.

\section{Materials and Methods}

\subsection{Study Population and Specimens}

Prostate tissue samples were obtained from men diagnosed with prostate cancer (prostatic adenocarcinoma) who underwent radical prostatectomies as part of their treatment. Prostate tissue samples were also obtained from men who underwent radical cystoprostatectomy for bladder cancer, with the prostate tissue subsequently confirmed to be non-malignant. Samples consisted of archival formalin-fixed paraffin-embedded (FFPE) punch biopsies sized $2 \times 2 \mathrm{~mm}$ (diameter $\times$ depth) obtained from men in the greater area of Melbourne, Australia, who had tissues submitted to TissuPath Pathology (Mount Waverley, Australia) for diagnostic pathology. Samples were analysed by a pathologist for the presence of malignant cells and were subsequently defined as being from benign or ma- 
lignant regions of the prostate. De-identified samples were received for HERV analysis and were analysed blinded. The study was approved by the Alfred Health Human Research and Ethics Committee (Project Number 32/11, approved on 28 March 2011).

\subsection{Cell Lines and Culture}

Primary prostate cancer cell lines DU145, LNCaP, PC3 (HTB-81, CRL-1740 and CRL1435 respectively, American Type Culture Collection (ATCC)), and the immortalised prostate cell line PNT1A (\#95012614, European Collection of Authenticated Cell Cultures), were generously provided by Renee Taylor and Gail Risbridger, Monash University, Clayton, Australia, and cultured at $37{ }^{\circ} \mathrm{C}$ in $5 \% \mathrm{CO}_{2}$ in Roswell Park Memorial Institute media (Gibco, Thermo Fisher Scientific, Waltham, MA, USA) containing 10\% foetal bovine serum, $2 \mathrm{mM}$ L-glutamine, $100 \mathrm{U} / \mathrm{mL}$ penicillin and $100 \mu \mathrm{g} / \mathrm{mL}$ of streptomycin (all from Thermo Fisher). The immortalised RWPE1 cell line (CRL-11609, ATCC) was cultured in keratinocyte serum-free media supplemented with $5 \mathrm{ng} / \mathrm{mL}$ human recombinant epidermal growth factor and $0.05 \mathrm{mg} / \mathrm{mL}$ bovine pituitary extract (Thermo Fisher). LNCaP, DU145 and PC3 cell lines are derived from prostate cancer cells and were isolated from a metastatic site (lymph node, brain and bone, respectively), whilst RWPE1 and PNT1A are normal prostate epithelial cells immortalised with HPV-18 and SV40 large T antigen, respectively.

\subsection{RNA Extraction and PCR Amplification of HERV Transcripts}

RNA was extracted from FFPE tissue using the High Pure FFPE RNA Micro-Isolation Kit (Roche Life Sciences, Penzberg, Germany), with modifications to improve yields including increasing the duration of proteinase $\mathrm{K}$ treatment from $30 \mathrm{~min}$ to overnight. RNA was extracted from $1 \times 10^{6}$ cultured prostate epithelial cells using the High Pure RNA Isolation Kit (Roche Life Sciences) as per the manufacturer's recommendations. Purified RNA was treated with RNase-free DNase I (Roche Life Sciences, Penzberg, Germany) to eliminate DNA carryover. cDNA was synthesized using the Transcriptor First-Strand cDNA Synthesis Kit (Roche Life Sciences) as per recommendations using random hexamers and anchored oligo (dT) primers. cDNA prepared from RNA extracted from normal primary human prostate epithelial cells was purchased from ScienCell Research Laboratories (Carlsbad, CA, USA).

The analysed HERV transcripts included four transcripts (HERV-K Gag, HERV-K Np9, HERV-W Gag and HERV-E Pol) previously reported to be upregulated in various cancers, including prostate cancer [19], and two additional transcripts, HERV-H Pol and HERV-E 4.1 surface protein (hereafter referred to as HERV-E Env), were identified through a comprehensive in silico analysis of putative HERV transcripts that possessed an intact LTR promoter, an ATG start codon and an open reading frame, and thus had the potential to express protein. HERV expression was normalised to housekeeping genes glyceraldehyde 3-phosphate dehydrogenase (GAPDH; using forward and reverse primers $5^{\prime}$-TGGTATCGTGGAAGGACTCATGAC-3' and $5^{\prime}$-ATGCCAGTGAGCTTCCCGTTCAGC$3^{\prime}$, respectively), TATA-box binding protein (TBP; using forward and reverse primers 5'-GAATATAATCCCAAGCGGTTTG-3' and 5'-ACTTCACATCACAGCTCCCC- $3^{\prime}$, respectively) and $18 \mathrm{~S}$ ribosomal RNA (18S rRNA; Applied Biosystems, Foster City, CA) by qPCR. Table 1 details the location and sequence of primers used to detect these transcripts and the number of amplicons predicted to exist within the reference human genome (hg38). The latter was determined by subjecting each primer pair to a specificity analysis using MFEprimer [20], using the default parameters except for annealing temperature $\left(55^{\circ} \mathrm{C}\right)$, size $(900 \mathrm{bp})$ and reference database (hg38.fa, interim_GRCh38.p10_rna.fa). 
Table 1. Details of human endogenous retrovirus (HERV) primers and genomic regions targeted.

\begin{tabular}{|c|c|c|c|c|c|}
\hline Target & $\begin{array}{c}\text { Amplicon } \\
\text { Size }\end{array}$ & Primers & Sequence $\left(5^{\prime}-3^{\prime}\right)$ & $\underset{1}{\text { Target Region or Reference }}$ & Predicted Targets ${ }^{2}$ \\
\hline HERV-K Gag & $113 \mathrm{bp}$ & $\begin{array}{l}\text { Fwd } \\
\text { Rev }\end{array}$ & $\begin{array}{l}\text { TTCCCGAGTACGTCTACAGTGA } \\
\text { GGTGTTTCTCATCAGGTGGAAT }\end{array}$ & [19] & 1 \\
\hline HERV-K Np9 & $113 \mathrm{bp}$ & $\begin{array}{l}\text { Fwd } \\
\text { Rev }\end{array}$ & $\begin{array}{l}\text { GTTAGTCTACAGGTGTATCCA } \\
\text { CTGTCTCTTTTCCCTACATTTCC }\end{array}$ & $\begin{array}{l}\text { GenBank Y17832.2 } \\
8836-8948\end{array}$ & 1 \\
\hline HERV-W Gag & $81 \mathrm{bp}$ & $\begin{array}{l}\text { Fwd } \\
\text { Rev }\end{array}$ & $\begin{array}{l}\text { TCAGGTCAACAATAGGATGACAACA } \\
\text { CAATGAGGGTCTACACTGGGAACT }\end{array}$ & [21] & 1 \\
\hline HERV-E Pol & $98 \mathrm{bp}$ & $\begin{array}{l}\text { Fwd } \\
\text { Rev }\end{array}$ & $\begin{array}{l}\text { CTCTACACAGTTAGGCTCG } \\
\text { GTGAAAATCCCCGCATAACC }\end{array}$ & $\begin{array}{c}\text { Chr13 } \\
40,349,095-40,349,192\end{array}$ & 1 \\
\hline HERV_E Env & $168 \mathrm{bp}$ & $\begin{array}{l}\text { Fwd } \\
\text { Rev }\end{array}$ & $\begin{array}{c}\text { ATTTGATGCTTGTGCAGCCA } \\
\text { TTCTTTTTCCAAGTAGCCCAAAT }\end{array}$ & [22] & $2^{3}$ \\
\hline HERV_H Pol & $118 \mathrm{bp}$ & $\begin{array}{l}\text { Fwd } \\
\text { Rev }\end{array}$ & $\begin{array}{l}\text { GCCAAACACATATACTCTCC } \\
\text { GAAGAGTGACTGGGATGAAG }\end{array}$ & $\begin{array}{c}\text { Chr8 } \\
8,066,673-8,066,790\end{array}$ & 1 \\
\hline
\end{tabular}

${ }^{1}$ Chromosome position based on Human Genome Sequence version hg18. ${ }^{2}$ Number of amplified targets predicted within the genome by MFEPrimer analysis. ${ }^{3}$ Two DNA targets are on chr17 and chr19, which are both 168 base pairs in length and share $95 \%$ nucleotide sequence identity.

cDNA was subjected to PCR using the Brilliant II SYBR Green qPCR Master Mix (Agilent Technologies, Santa Clara, CA) and the following thermocycling parameters on an Mx3000P qPCR machine (Agilent Technologies): initial denaturation step at $95^{\circ} \mathrm{C}$ for $10 \mathrm{~min}, 40$ amplification cycles at $95^{\circ} \mathrm{C}$ for $30 \mathrm{~s}$ and $60^{\circ} \mathrm{C}$ for $1 \mathrm{~min}$, followed by melt-curve analysis. The qPCR analysis for all transcripts was performed in triplicate and HERV expression was normalized to housekeeping gene copy number. Plasmid DNA standards were generated for each HERV target by cloning the specific PCR amplicon into a pTOPO cloning vector and amplicon integrity and accuracy were confirmed by DNA sequencing. Serial dilutions of plasmid standards were generated and included in each assay.

\subsection{Detection of HERV-K Gag Protein by Immunohistochemistry}

The presence of HERV-K Gag protein in matched benign and malignant prostate tissue was assessed in FFPE biopsy material from 18 randomly selected participants using immunohistochemistry (IHC). Three micron-tissue sections were cut from selected blocks and stored at $4{ }^{\circ} \mathrm{C}$ in desiccated containers until used. IHC was performed using the Leica BOND-Max autostainer (Leica Biosystems, Buffalo Grove, IL, USA). Sections were stained with supernatant from the TI-35 mouse hybridoma raised against HERV-K Gag protein [16] (generously supplied by Prof Eiichi Nakayama, Kawasaki University Medical Welfare, Kurashiki, Japan) for $30 \mathrm{~min}$ and detected with the BOND Polymer refine detection kit (Leica Biosystems) with 1/100 dilution and ER2 retrieval for $20 \mathrm{~min}$. Staining was assessed on blinded samples by one of the authors (J.P.), an anatomical pathologist specializing in urological cancers. HERV-K Gag expression was scored individually for benign and malignant sections as either negative or positive, with an overall staining intensity of weak $(+)$, moderate $(++)$, or strong $(+++)$.

\section{Results}

3.1. Expression of HERV Transcripts Is Significantly Upregulated in Prostate Cancer Cell Lines as Compared to Normal Epithelial Cells

We determined the expression pattern of six HERV transcripts in cDNA derived from primary prostate epithelial cells and three primary and two immortalised prostate cancer cell lines using $\mathrm{qPCR}$, standardising expression relative to the housekeeping gene GAPDH. This analysis revealed a pattern of over-expression of most HERV transcripts in the majority of prostate cancer cell lines, with some variations. HERV-K Gag expression was significantly and specifically upregulated in LNCap and PC3 cells lines (Figure 1A), whilst HERV-K Np9, HERV-W Gag, HERV-H Pol and HERV-E Pol expression were upregulated in almost all cell lines tested as compared to normal epithelial cells (Figure 1B-E). HERV-E Env expression was more variable, showing a downregulation in DU154 and PNT1A cell lines but an upregulation in LNCaP (Figure 1F).

To address the possibility that malignant and transformed cells exhibit altered housekeeping gene expression, we also standardised HERV expression to two other housekeeping 
genes, 18S rRNA and TBP (Supplementary Figure S1). A similar pattern of HERV transcript overexpression was largely observed for HERV-K Gag, -E Pol and -W Gag transcripts, irrespective of the gene used for standardisation. Patterns of expression of HERV-H Pol, -E Env and -K Np9 transcripts relative to normal epithelial cells were also consistent when standardised to TBP, although a different expression profile for these genes was seen when $18 \mathrm{~S}$ rRNA was used for standardisation. Taken together, these data are consistent with an altered transcriptional state in malignant and transformed prostate epithelial cells, which results in increased expression of a range of HERV transcripts.

A

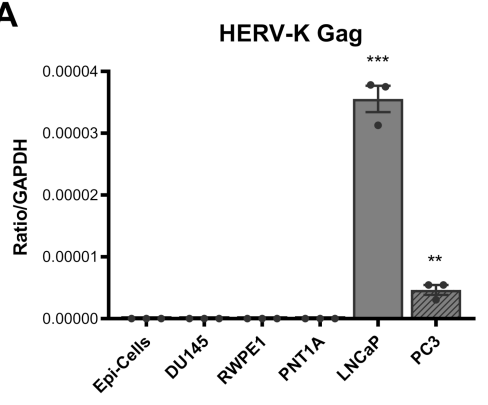

D

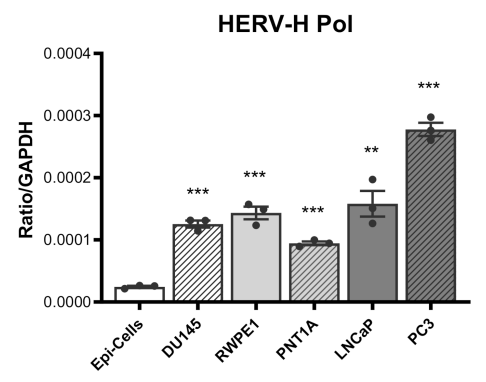

B

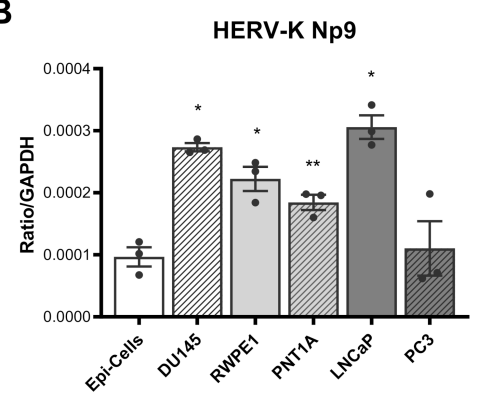

E

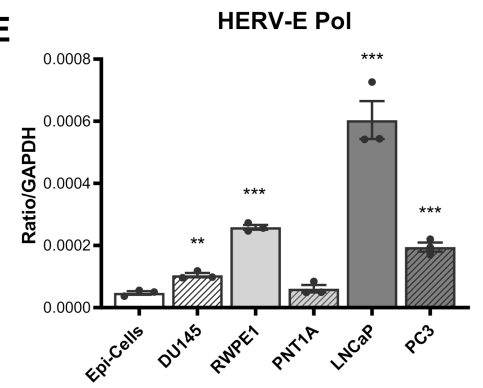

C

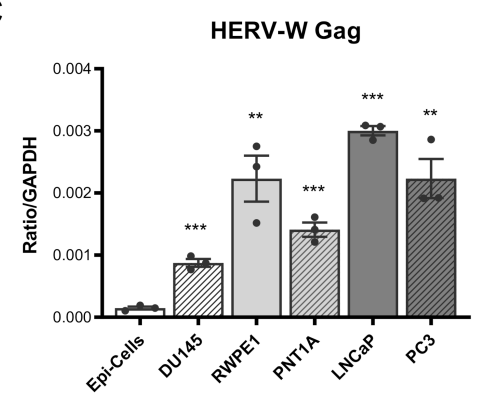

$\mathbf{F}$

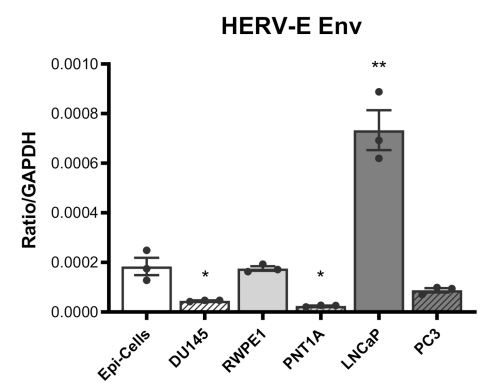

Figure 1. HERV transcript expression in prostate cancer cell lines and primary prostate epithelial cells. Expression of HERVK Gag (A), HERV-K Np9 (B), HERV-W Gag (C), HERV-H Pol (D), HERV-E Pol (E) and HERV-E Env (F) transcripts were detected in cDNA synthesised from RNA purified from primary non-cancerous prostate epithelial cells (Epi-cells), DU145, RWPE1, PNT1A, LNCaP and PC3 cells by qPCR. Copies of HERV transcripts were standardised to the house-keeping gene GAPDH detected within the same samples. Graphs show the mean and standard error of the mean of results from $n=3$ independent experiments. ${ }^{*}, * *$, and ${ }^{* * *}$ indicate $p<0.05,0.01$, and 0.001 respectively, compared to primary epithelial cells, as determined by Student's unpaired $t$-test.

3.2. HERV-K Gag Expression Is Significantly Upregulated in Malignant, but Not Benign, Regions of Primary Prostate Tissue

To investigate whether expression of HERV transcripts is altered in primary prostate cancer tissue, we analysed matched biopsy samples of both benign and malignant regions of the prostate taken from men diagnosed with prostate cancer (prostatic adenocarcinoma) and compared data with expression patterns observed in prostate biopsies taken from men without prostate malignancy. Prostate tissue samples were obtained from 27 males diagnosed with prostate cancer who had radical prostatectomies, with a median age of 65.2 (range: 47-70). The median Gleason score of prostate cancer donors was 7 (range: 6 to 9), with $2(7.4 \%), 18(66.7 \%), 4(14.8 \%)$ and $3(11.1 \%)$ donors having Gleason scores of $6,7,8$ and 9 (out of a possible 10), respectively. The proportion of participants with Gleason score of 8-10 (representing high-grade cancers) in this study was therefore $25.9 \%$.

Given the variation in housekeeping gene expression observed in prostate epithelial cell lines, we first compared expression of the housekeeping genes GAPDH, 18S rRNA and TBP in the primary tissue biopsies to identify the most robust gene to use for normalisation. The expression of TBP was typically lower than that of GAPDH and 18S rRNA and 
was undetectable in many biopsy samples. Whilst comparable levels of $18 \mathrm{~S}$ rRNA and GAPDH were detected per mg of tissue weight, GAPDH was more consistently detected in tissue samples and expression levels did not differ due to malignancy status. Thus, in subsequent analyses, GAPDH was utilised to standardise expression of HERV genes in primary tissue samples.

Analysis of HERV transcripts in primary tissue biopsy samples from prostate cancerfree males indicated that the HERV transcripts examined here were expressed at low but detectable levels in these individuals, with the exception of HERV-K Gag, which was only detected in $55 \%$ of samples tested (Figure 2A). Next, we compared HERV transcript expression in these prostate cancer-free individuals with samples taken from benign regions of the prostate in individuals with prostate cancer and found significantly upregulated expression of HERV-E Pol ( $p=0.003$, Figure 2E) and a trend towards increased expression of HERV-K Np9 ( $p=0.054$, Figure 2B), but decreased expression of HERV-E Env $(p<0.001$, Figure $2 \mathrm{~F}$ ) in men with prostate cancer as compared to those without. The changes to HERV-E Env and Pol expression were also observed in malignant regions of the prostate, suggesting global transcriptional alternations within prostate tissues not specific to the malignant cells themselves.

A

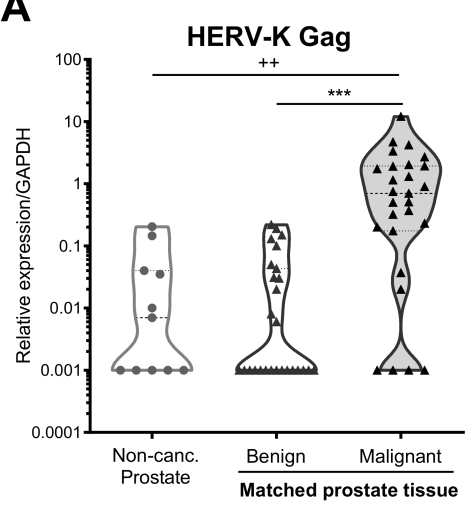

D

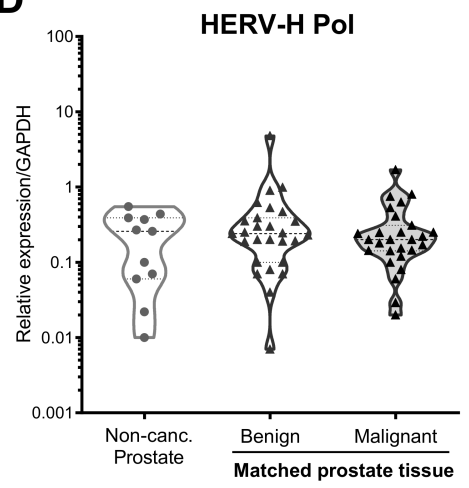

B

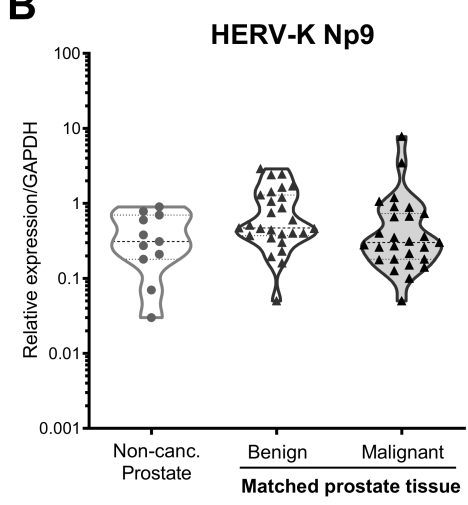

E

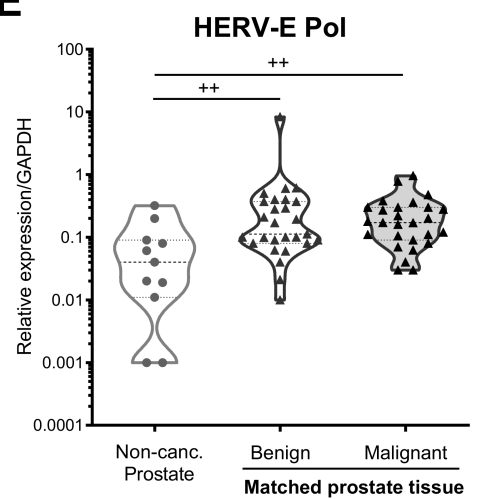

C

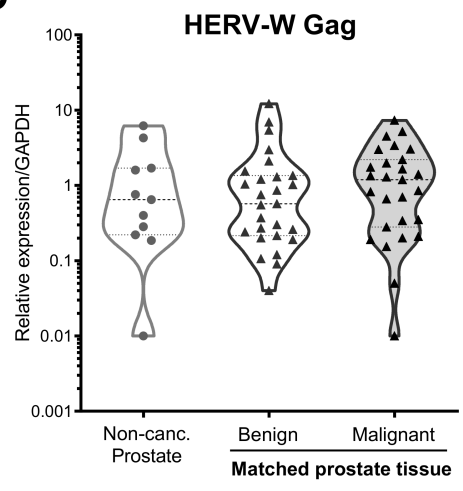

F

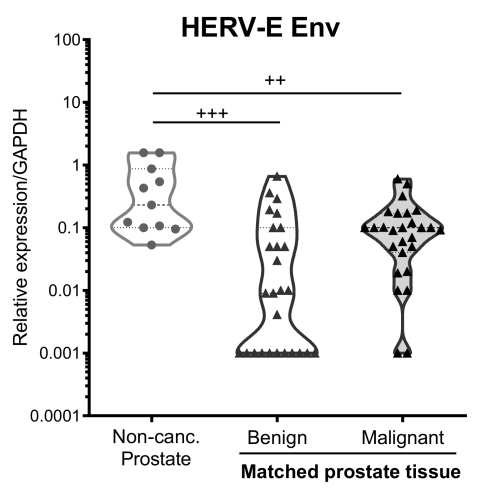

Figure 2. HERV transcript expression in prostate biopsy samples from males with and without prostate cancer. Expression of HERV-K Gag (A), HERV-K Np9 (B), HERV-W Gag (C), HERV-H Pol (D), HERV-E Pol (E) and HERV-E Env (F) transcripts were detected in RNA extracted from punch biopsy prostate samples from men without prostate cancer (Non-Canc. Prostate, $n=11)$, or from matched benign and malignant regions of the prostate from individuals with prostate cancer $(n=27)$ by qRT-PCR. Graphs show truncated violin plots of the average gene expression standardised to copies of GAPDH from $n=3$ replicates per sample. Samples with undetectable levels of HERV transcript are shown with a value of 0.001 for the purposes of visualisation in the graphs, and the actual value of 0 was used for statistical analyses. ++ , and +++ indicate $p<0.01$ and 0.001 respectively, as compared to non-cancerous prostate tissue, determined by Mann-Whitney U-test. ${ }^{* * *}$ indicates $p<0.001$ for comparison of matched benign and malignant tissue, as determined by Wilcoxon matched-pairs signed-rank test. Non-parametric tests were used due to non-normal distribution of the data. 
Comparison of HERV expression levels between matched benign and malignant regions within the prostate of men with prostate cancer revealed a striking upregulation of HERV-K Gag expression in malignant regions of the prostate $(p<0.001$, Figure $2 \mathrm{~A})$, with $23 / 27(85.2 \%)$ of donors analysed showing increased HERV-K Gag expression in malignant regions (Supplementary Figure S2). There were no significant differences in expression of any other HERV transcripts analysed between benign and malignant regions of the prostate, although all of the 11 individuals with undetectable HERV-E Env expression in benign samples had detectable expression in malignant regions (Supplementary Figure S2F). Taken together, these data indicate that in this cohort, prostate cancer was associated with global changes in transcription of certain HERV elements in both benign and malignant prostate epithelial cells, and with a significant upregulation of HERV-K Gag expression exclusively in malignant cells.

\subsection{Analysis of the Relationship between the Expression of Different HERV Transcripts in Malignant Regions of the Prostate}

Whilst HERV-K Gag was the only transcript analysed which showed significantly altered expression in malignant tissues as compared to matched benign regions at a cohort level, a number of individuals exhibited malignancy-associated alterations to expression of a range of HERV transcripts (Figure 3A). We therefore analysed the association between HERV-K Gag transcript upregulation and other HERV transcripts in men with prostate cancer and found a significant positive association between expression of HERV-K Gag and all other HERV transcripts, except HERV-K Np9 (Figure 3B, Spearman's rho: 0.39$0.46, p<0.05$ for all). Interestingly, HERV-K Np9 expression itself showed a significant positive association with expression of the same transcripts (rho: $0.42-0.54, p<0.05$ for all). Significant associations were also observed between expression of HERV-E Env and HERV-H Pol (rho: 0.58, $p=0.001$ ), and HERV-E Pol and HERV-W Gag (rho: 0.55, $p=0.003$ ). We further investigated whether there was any relationship between HERV expression and age or Gleason score, but did not observe any significant associations. These data are consistent with an altered transcriptional profile in malignant prostate epithelial cells that leads to over-expression of a range of different HERV transcripts, but the magnitude of this upregulation shows substantial inter-individual variation.

\subsection{HERV-K Gag Protein Is Detected Primarily in Malignant Regions of the Prostate in Men with} Prostate Cancer

Whilst malignancy-associated changes to the transcriptional profile of cells may lead to elevated levels of HERV RNAs, the heavily mutated nature of HERV sequences in the genome may preclude the translation of these sequences into proteins. We therefore performed immunohistochemical staining of biopsy sections from 18 of the 27 study participants to explore whether expression of HERV protein was also upregulated in malignant prostate tissue. For this analysis, we focused on HERV-K Gag given the significant upregulation of this transcript in the majority of participants in this study. Positive immunohistochemical staining of HERV-K Gag protein was detected in 12/18 (66.7\%) malignant tissues, with 8 of these 12 positive samples showing areas with medium- to high-intensity staining, but only $1 / 18$ (5.6\%) benign tissue sections (Table 2 and Figure 4). Furthermore, comparison of immunohistochemistry results with the extent of transcript expression indicated a trend towards detection of HERV-K Gag protein in samples which showed a greater over-expression of RNA transcript (Table 2). These data indicate that malignancy in prostate epithelial cells is associated with increased transcription and translation of HERV-K Gag sequences and that the detection of HERV-K Gag protein may be a useful discriminatory biomarker of malignancy in this setting. 
A

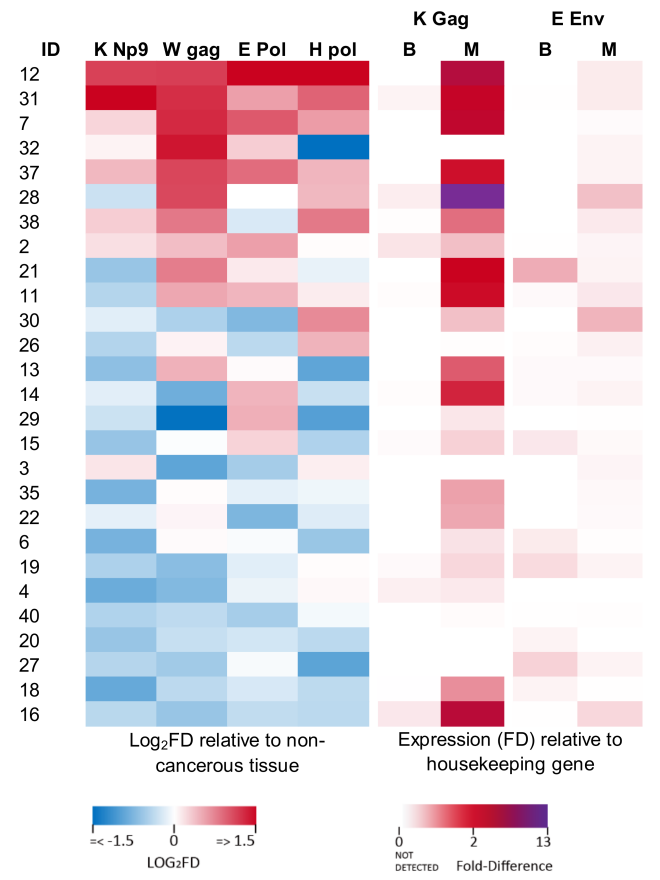

B

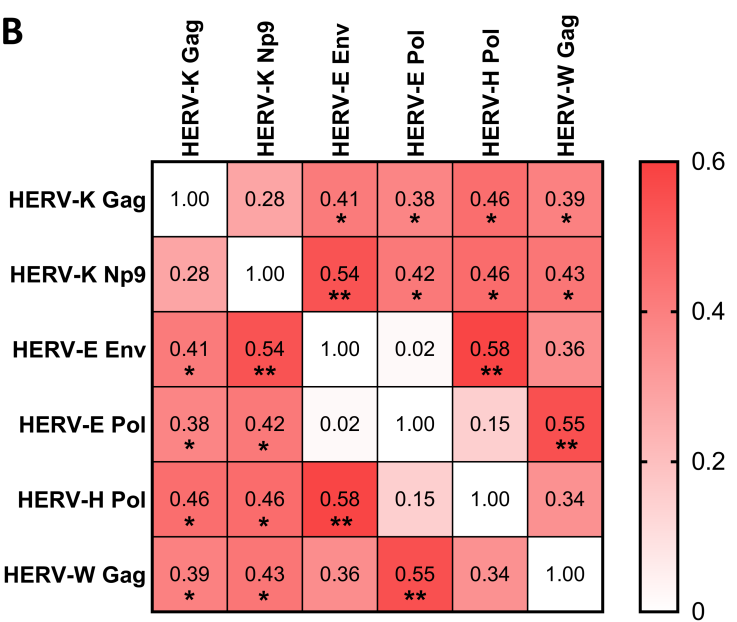

Figure 3. Associations between expression of various HERV transcripts in prostate cancer tissue. (A) Heat map showing the differential expression ( $\log _{2}$ fold difference; FD) of HERV RNA transcripts in prostate cancer tissues from men with prostate cancer. Left panels show expression of HERV-K Np9, HERV-W Gag, HERV-E Pol and HERV-H Pol in malignant regions relative to benign regions of the prostate (each standardised to GAPDH). Right panels show expression of HERV-K Gag and HERV-E Env in both benign (B) and malignant (M) regions relative to expression of GAPDH (due to transcripts being undetectable in many benign tissue samples). (B) Correlation matrix showing the association between expression levels of the various HERV transcripts as determined by non-parametric Spearman's correlation. For HERV-K Gag and HERV-E Env, the expression level in malignant tissues (relative to GAPDH) was analysed, whilst for all other transcripts, the expression in malignant tissue relative to benign tissue (both standardised to GAPDH) was used. Values show Spearman's rho. * and ** indicate $p<0.05$ and 0.01 , respectively.

Table 2. Detection of HERV-K Gag protein in malignant and benign prostate tissue from men with prostate cancer.

\begin{tabular}{|c|c|c|c|c|c|c|}
\hline \multirow[t]{2}{*}{ Sample ID } & \multirow[t]{2}{*}{ Age } & \multirow[t]{2}{*}{ Gleason Score } & \multicolumn{2}{|c|}{ HERV K Gag Detected by IHC ${ }^{1}$} & \multicolumn{2}{|c|}{ HERV K Gag Detected by qRT-PCR } \\
\hline & & & Benign & Cancerous & Benign & Cancerous \\
\hline 71 & 59 & 7 & - & $+/++$ & ND & 12.3 \\
\hline 72 & 66 & 7 & - & $+/++^{2}$ & ND & 1.2 \\
\hline 74 & 53 & 7 & - & - & ND & ND \\
\hline 75 & 65 & 7 & $-{ }^{3}$ & - & ND & ND \\
\hline 76 & 62 & 7 & - & + & ND & ND \\
\hline 77 & 47 & 8 & - & - & 0.15 & 12.1 \\
\hline 78 & 61 & 6 & $-^{3}$ & + & ND & 0.2 \\
\hline 79 & 69 & 7 & - & $++/+++$ & ND & 0.5 \\
\hline 80 & 65 & 7 & - & - & ND & 2.7 \\
\hline 83 & 54 & 7 & - & $+/+++$ & ND & ND \\
\hline 84 & 65 & 7 & - & -3 & 0.4 & 1.2 \\
\hline 85 & 66 & 7 & - & $+/++$ & ND & 10.1 \\
\hline 86 & 65 & 8 & - & - & ND & 0.75 \\
\hline 87 & 69 & 7 & -3 & + & 0.01 & 4.89 \\
\hline 88 & 70 & 9 & - & $++/+++$ & ND & 1.9 \\
\hline 94 & 66 & 8 & $t^{2}$ & + & 0.02 & 1.15 \\
\hline 97 & 65 & 8 & - & $++/+++$ & ND & ND \\
\hline 98 & 64 & 7 & - & ++ & ND & 0.04 \\
\hline
\end{tabular}

${ }^{1}$ Samples were graded as having no staining (-) or low-, medium- and high-level staining $(+,++$ or +++ , respectively) by a single experienced Pathologist. ${ }^{2}$ Variable or focal staining observed. ${ }^{3}$ Some positive staining in atrophic areas. ${ }^{4}$ Corresponding expression level of HERV-K Gag mRNA (standardised to GAPDH) for each sample is shown for comparison. IHC: immunohistochemistry, ND: not detected. 


\section{A: Benign}

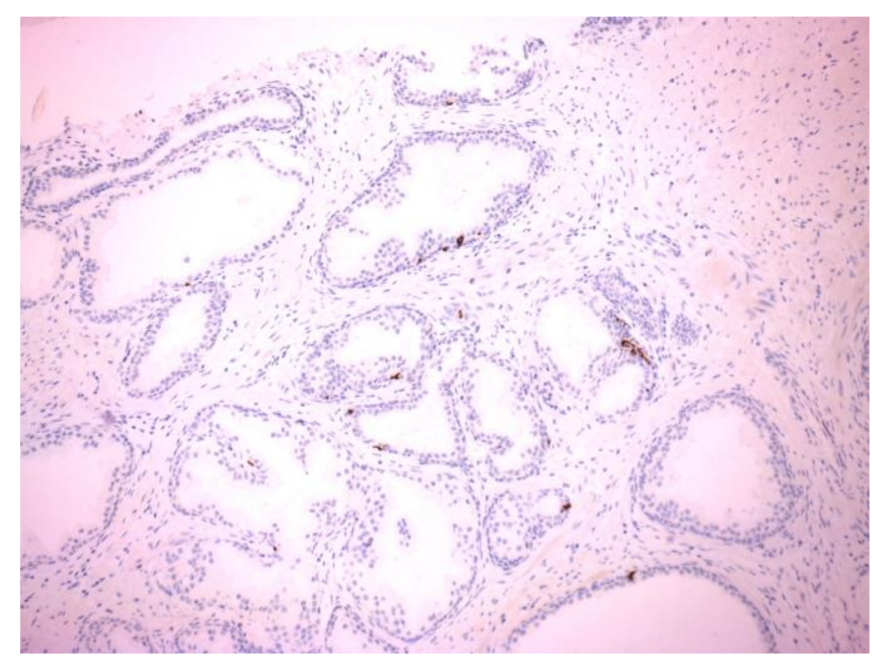

B: Malignant

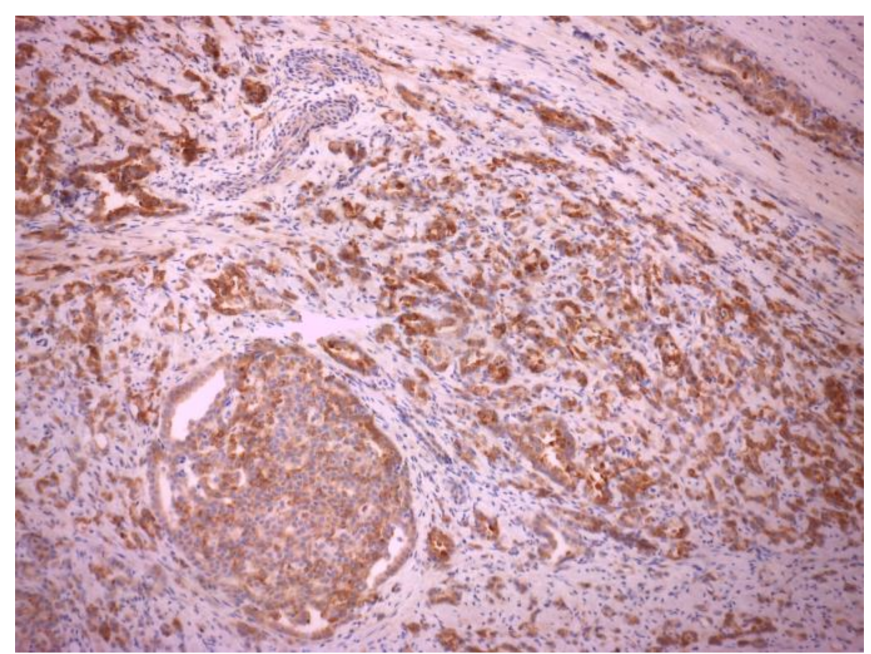

Figure 4. Expression of HERV-K Gag protein in cancerous regions of prostate tissue sections. HERV-K Gag protein was detected in sections of matched malignant and benign regions of formalin-fixed paraffin-embedded prostate biopsy tissue from 18 males with prostate cancer by immunohistochemistry. Immunohistochemical images from benign prostate tissue (A) or malignant prostatic adenocarcinoma (B) regions of the prostate showing negative and positive staining for HERV-K Gag protein from one representative individual are shown.

\section{Discussion}

Malignancy-associated changes in the expression of HERV elements are associated with clinically relevant outcomes in a range of cancer settings and may potentially represent a therapeutic target to specifically identify and target malignant cells. Here, we used matched cancerous and benign tissues from prostate cancer-affected men to demonstrate the specific upregulation of HERV-K Gag RNA and protein within malignant regions of the prostate, highlighting the potential utility of this biomarker to be used for diagnostic, prognostic and therapeutic applications. The finding that heightened expression of HERV-K Gag was restricted to malignant cells suggests that it may be the result of intrinsic cellular changes, such as epigenetic alterations or the action of cell-associated oncogenic factors. We also found significantly elevated expression of HERV-K Gag mRNA in the prostate cancer cell line LNCaP, and to a lesser extent in PC-3 cells, which is consistent with previous findings in some studies [19] but not others [16]. We utilised primers recognising the HERV-K101 provirus but dozens of different HERV-K proviruses have been documented in the human genome with different prostate cancer cell lines showing varied expression of certain loci [23]. This, combined with differences in target amplicons analysed, may influence the expression profiles detected in various studies and highlights that HERV expression profiles are highly sequence- and cell-specific.

Our findings of increased HERV-K Gag mRNA and protein expression in malignant prostate cancerous cells are consistent with the findings of others who showed increased expression of HERV-K Gag mRNA in prostate cancer tissue $[15,16]$ and the detection of HERV-K Gag protein in $\approx 85 \%$ of prostate cancer tissues as compared to approximately $\approx 40 \%$ of normal prostate tissues [15]. Wallace et al. reported elevated levels of HERV-K mRNA not only in prostate tissue from men with prostate cancer but also in PBMC [18], highlighting the potential prognostic utility of this low-invasive parameter. Interestingly, this latter study also identified a significant effect of both race and age on the association between HERV-K element expression and prostate cancer, emphasising the need to validate such biomarkers in the intended target population. Although at a cohort level we did not see a significant difference in expression of HERV-E Env transcripts in malignant as compared to benign prostate tissue, 11 individuals (40.7\%) exhibited undetectable levels of HERV-E Env in benign tissue and all of these showed elevated transcript levels in 
malignant tissue. This is similar to the observations of Wang-Johanning et al. [17], who showed detectable levels of HERV-E Env mRNA in 38.8\% of prostate tissues from cancerous patients compared to $5.6 \%$ of normal prostate tissue.

It remains unclear whether the HERV-derived elements play a role in initiating or enhancing malignancy, or whether they are produced as a consequence of epigenetic alterations to cancerous cells. HERV LTRs can act as alternative promoters for host gene expression and modulate expression of genes, including oncogenes and proto-oncogenes (see Reference [24] for a recent review). Whilst the association of HERV overexpression with global DNA hypomethylation in cancer supports the idea that HERV expression is secondary to malignant changes [19], there is evidence that HERV nucleic acids and proteins can potentiate malignancy, with HERV-K Rec and Np9 proteins recognised as proto-oncogenes [25]. Furthermore, HERVs contain an immunosuppressive element which can modify the cytokine response from PBMC, which has been shown in mouse models to induce the recruitment of immunosuppressive cells to the tumour microenvironment [26]. Expression of HERV-H RNA has also been implicated in tumour invasion and metastases both in vitro and in vivo [26], suggesting that in some settings, HERVs may play an active role in potentiating malignant processes.

Whilst HERV elements have been evaluated as potential targets for adjunct therapies with encouraging results (reviewed in Reference [24]), their most promising role is as diagnostic or prognostic biomarkers of malignancy. In this regard, there is ample evidence linking heightened HERV element expression with clinical outcomes such as metastases, disease severity and mortality in a range of cancer settings, including breast [14,27], melanoma [28] and germ cell tumours [29]. Specifically, in prostate cancers, Reis et al. found that the presence of serum antibodies to HERV-K Gag was almost exclusively restricted to individuals with more severe disease, and those with detectable antibodies tended to have poorer disease outcomes [15]. These findings support a potential role for HERV-K Gag as an adjunct diagnostic marker for prostate cancer, similar to that demonstrated for breast cancer, where HERV-K Gag antibodies and mRNA were found to be a sensitive and specific marker of early-stage cancers [13]. The relationship between expression levels of HERV mRNA, protein and serum antibodies in various malignancies, and which biomarker has the most useful prognostic value, remains to be fully defined.

This study had a number of important limitations, including its relatively small sample size and cross-sectional design, which precluded the analysis of clinical outcomes associated with increased HERV expression. It would therefore be of significant interest to confirm these findings in a longitudinal study of men with a broader range of prostate cancer severities with well-characterised clinical outcomes, to confirm the potential value of HERV over-expression as a useful prognostic marker. Evidence of an association between HERV expression and more severe or progressive prostate cancer may indicate a potential benefit of screening for HERV expression at diagnosis or during ongoing monitoring to inform decisions regarding the need for surgical interventions. Given the ability to detect HERV elements in blood, and the limited but enticing data associating its detection with more severe disease, future work should explore the relationship between HERV expression in tissue and blood compartments to determine whether a minimally invasive blood test could identify cellular changes occurring in prostate tissue. The potential utility of HERV elements as a screening tool or prognostic biomarker to be used in combination with PSA testing to identify high-risk cancers offers the opportunity to further improve prostate cancer prevention and minimise over-treatment of this common cancer.

Supplementary Materials: The following are available online at https:/ / www.mdpi.com/1999-491 5/13/3/449/s1, Figure S1: HERV RNA expression in cell lines and primary prostate epithelial cells, Figure S2: HERV transcript expression in benign and malignant regions of the prostate in men with prostate cancer. 
Author Contributions: Conceptualisation, G.T., A.C.H. and J.M.; methodology, S.D.R., A.C.H. and J.A.H.; validation, S.N.; formal analysis, S.D.R. and A.C.H.; investigation, S.D.R. and J.P.; resources, G.T. and J.M.; writing — original draft preparation, A.C.H. and S.D.R.; writing-review and editing, J.A.H. and G.T.; supervision, G.T.; project administration, S.D.R.; funding acquisition, G.T. All authors have read and agreed to the published version of the manuscript.

Funding: This research was supported by the Prostate Cancer Foundation of Australia (PCFA) grant CG 0710 awarded to G.T., and G.T. was also supported by the National Health and Medical Research Council of Australia (NHMRC) Senior Research Fellowship GNT1117748. We gratefully acknowledge the contribution to this work of the Victorian Operational Infrastructure Support Program received by the Burnet Institute.

Institutional Review Board Statement: The study was conducted according to the guidelines of the Declaration of Helsinki and approved by the Ethics Committee of the Alfred Hospital, Melbourne, Australia (Project 32/11, approved on 28 March 2011).

Informed Consent Statement: Informed consent was obtained from all subjects involved in the study. Data Availability Statement: Not applicable.

Acknowledgments: We thank Eiichi Nakayama (Kawasaki University of Medical Welfare, Japan) for the generous donation of the anti-HERV-K antibody.

Conflicts of Interest: The authors declare no conflict of interest. The funders had no role in the design of the study; in the collection, analyses, or interpretation of data; in the writing of the manuscript, or in the decision to publish the results.

\section{References}

1. Australian Institute of Health and Welfare. Cancer Data in Australia. Available online: https://www.aihw.gov.au/reports/ cancer/cancer-data-in-australia/contents / cancer-summary-data-visualisation (accessed on 12 February 2021).

2. Prostate Cancer Foundation of Australia. Prostate Cancer in Australia-What Do the Numbers Tell Us? Available online: https://www.prostate.org.au/news-media/news/prostate-cancer-in-australia-what-do-the-numbers-tell-us/ (accessed on 12 February 2021).

3. Glasziou, P.P.; Jones, M.A.; Pathirana, T.; Barratt, A.L.; Bell, K.J. Estimating the magnitude of cancer overdiagnosis in Australia. Med. J. Aust. 2020, 212, 163-168. [CrossRef]

4. Geis, F.K.; Goff, S.P. Silencing and transcriptional regulation of endogenous retroviruses: An overview. Viruses 2020, $12,884$. [CrossRef] [PubMed]

5. Buscher, K.; Hahn, S.; Hofmann, M.; Trefzer, U.; Ozel, M.; Sterry, W.; Lower, J.; Lower, R.; Kurth, R.; Denner, J. Expression of the human endogenous retrovirus-K transmembrane envelope, Rec and Np9 proteins in melanomas and melanoma cell lines. Melanoma Res. 2006, 16, 223-234. [CrossRef] [PubMed]

6. Schulte, A.M.; Lai, S.; Kurtz, A.; Czubayko, F.; Riegel, A.T.; Wellstein, A. Human trophoblast and choriocarcinoma expression of the growth factor pleiotrophin attributable to germ-line insertion of an endogenous retrovirus. Proc. Natl. Acad. Sci. USA 1996, 93, 14759-14764. [CrossRef] [PubMed]

7. Jern, P.; Coffin, J.M. Effects of retroviruses on host genome function. Ann. Rev. Genet. 2008, 42, 709-732. [CrossRef]

8. Grabski, D.F.; Hu, Y.; Sharma, M.; Rasmussen, S.K. Close to the bedside: A systematic review of endogenous retroviruses and their impact in oncology. J. Surg. Res. 2019, 240, 145-155. [CrossRef]

9. Levet, S.; Charvet, B.; Bertin, A.; Deschaumes, A.; Perron, H.; Hober, D. Human endogenous retroviruses and type 1 diabetes. Curr. Diab. Rep. 2019, 19, 141. [CrossRef]

10. Wang-Johanning, F.; Radvanyi, L.; Rycaj, K.; Plummer, J.B.; Yan, P.; Sastry, K.J.; Piyathilake, C.J.; Hunt, K.K.; Johanning, G.L. Human endogenous retrovirus $\mathrm{K}$ triggers an antigen-specific immune response in breast cancer patients. Cancer Res. 2008, 68, 5869-5877. [CrossRef] [PubMed]

11. Rycaj, K.; Plummer, J.B.; Yin, B.; Li, M.; Garza, J.; Radvanyi, L.; Ramondetta, L.M.; Lin, K.; Johanning, G.L.; Tang, D.G.; et al. Cytotoxicity of human endogenous retrovirus K-specific T cells toward autologous ovarian cancer cells. Clin. Cancer Res. 2015, 21, 471-483. [CrossRef] [PubMed]

12. Ma, W.; Hong, Z.; Liu, H.; Chen, X.; Ding, L.; Liu, Z.; Zhou, F.; Yuan, Y. Human endogenous retroviruses-K (HML-2) expression is correlated with prognosis and progress of hepatocellular carcinoma. Biomed. Res. Int. 2016, 2016, 8201642. [CrossRef]

13. Wang-Johanning, F.; Li, M.; Esteva, F.J.; Hess, K.R.; Yin, B.; Rycaj, K.; Plummer, J.B.; Garza, J.G.; Ambs, S.; Johanning, G.L. Human endogenous retrovirus type $\mathrm{K}$ antibodies and mRNA as serum biomarkers of early-stage breast cancer. Int. J. Cancer 2014, 134, 587-595. [CrossRef] [PubMed]

14. Golan, M.; Hizi, A.; Resau, J.H.; Yaal-Hahoshen, N.; Reichman, H.; Keydar, I.; Tsarfaty, I. Human endogenous retrovirus (HERV-K) reverse transcriptase as a breast cancer prognostic marker. Neoplasia 2008, 10, 521-533. [CrossRef] 
15. Reis, B.S.; Jungbluth, A.A.; Frosina, D.; Holz, M.; Ritter, E.; Nakayama, E.; Ishida, T.; Obata, Y.; Carver, B.; Scher, H.; et al. Prostate cancer progression correlates with increased humoral immune response to a human endogenous retrovirus GAG protein. Clin. Cancer Res. 2013, 19, 6112-6125. [CrossRef]

16. Ishida, T.; Obata, Y.; Ohara, N.; Matsushita, H.; Sato, S.; Uenaka, A.; Saika, T.; Miyamura, T.; Chayama, K.; Nakamura, Y.; et al. Identification of the HERV-K gag antigen in prostate cancer by SEREX using autologous patient serum and its immunogenicity. Cancer Immun. 2008, 8, 15.

17. Wang-Johanning, F.; Frost, A.R.; Jian, B.; Azerou, R.; Lu, D.W.; Chen, D.T.; Johanning, G.L. Detecting the expression of human endogenous retrovirus E envelope transcripts in human prostate adenocarcinoma. Cancer 2003, 98, 187-197. [CrossRef]

18. Wallace, T.A.; Downey, R.F.; Seufert, C.J.; Schetter, A.; Dorsey, T.H.; Johnson, C.A.; Goldman, R.; Loffredo, C.A.; Yan, P.; Sullivan, F.J.; et al. Elevated HERV-K mRNA expression in PBMC is associated with a prostate cancer diagnosis particularly in older men and smokers. Carcinogenesis 2014, 35, 2074-2083. [CrossRef]

19. Goering, W.; Ribarska, T.; Schulz, W.A. Selective changes of retroelement expression in human prostate cancer. Carcinogenesis 2011, 32, 1484-1492. [CrossRef]

20. Wang, K.; Li, H.; Xu, Y.; Shao, Q.; Yi, J.; Wang, R.; Cai, W.; Hang, X.; Zhang, C.; Cai, H.; et al. MFEprimer-3.0: Quality control for PCR primers. Nucleic Acids Res. 2019, 47, W610-W613. [CrossRef]

21. Nellaker, C.; Yao, Y.; Jones-Brando, L.; Mallet, F.; Yolken, R.H.; Karlsson, H. Transactivation of elements in the human endogenous retrovirus W family by viral infection. Retrovirology 2006, 3, 44. [CrossRef]

22. Andersson, A.C.; Yun, Z.; Sperber, G.O.; Larsson, E.; Blomberg, J. ERV3 and related sequences in humans: Structure and RNA expression. J. Virol. 2005, 79, 9270-9284. [CrossRef] [PubMed]

23. Agoni, L.; Guha, C.; Lenz, J. Detection of human endogenous retrovirus K (HERV-K) transcripts in human prostate cancer cell lines. Front. Oncol. 2013, 3, 180. [CrossRef]

24. Curty, G.; Marston, J.L.; de Mulder Rougvie, M.; Leal, F.E.; Nixon, D.F.; Soares, M.A. Human endogenous retrovirus K in cancer: A potential biomarker and immunotherapeutic target. Viruses 2020, 12, 726. [CrossRef] [PubMed]

25. Denne, M.; Sauter, M.; Armbruester, V.; Licht, J.D.; Roemer, K.; Mueller-Lantzsch, N. Physical and functional interactions of human endogenous retrovirus proteins Np9 and rec with the promyelocytic leukemia zinc finger protein. J. Virol. 2007, 81, 5607-5616. [CrossRef]

26. Kudo-Saito, C.; Yura, M.; Yamamoto, R.; Kawakami, Y. Induction of immunoregulatory CD271+ cells by metastatic tumor cells that express human endogenous retrovirus H. Cancer Res. 2014, 74, 1361-1370. [CrossRef]

27. Zhao, J.; Rycaj, K.; Geng, S.; Li, M.; Plummer, J.B.; Yin, B.; Liu, H.; Xu, X.; Zhang, Y.; Yan, Y.; et al. Expression of human endogenous retrovirus type $\mathrm{K}$ envelope protein is a novel candidate prognostic marker for human breast cancer. Genes Cancer 2011, 2, 914-922. [CrossRef]

28. Hahn, S.; Ugurel, S.; Hanschmann, K.M.; Strobel, H.; Tondera, C.; Schadendorf, D.; Lower, J.; Lower, R. Serological response to human endogenous retrovirus $\mathrm{K}$ in melanoma patients correlates with survival probability. AIDS Res. Hum. Retrovir. 2008, 24, 717-723. [CrossRef]

29. Kleiman, A.; Senyuta, N.; Tryakin, A.; Sauter, M.; Karseladze, A.; Tjulandin, S.; Gurtsevitch, V.; Mueller-Lantzsch, N. HERVK(HML-2) GAG/ENV antibodies as indicator for therapy effect in patients with germ cell tumors. Int. J. Cancer 2004, 110, 459-461. [CrossRef] 